\title{
A Variable Stiffness Gripper with Antagonistic Magnetic Springs for Enhancing Manipulation
}

\author{
Amirhossein H. Memar and Ehsan T. Esfahani \\ Department of Mechanical and Aerospace Engineering, University at Buffalo \\ Email: < ahajiagh, ehsanesf $>$ @ buffalo.edu
}

\begin{abstract}
Robot grasping of objects based on variable stiffness actuation not only improves the safety and robustness of the grasp but also enhances dynamic manipulation. In this paper, we present the design aspects of a variable stiffness gripper and demonstrate how the controllable compliance of the fingers can improve the performance in dynamic manipulation tasks such as hammering/hitting. The proposed gripper consists of two parallel fingers and repulsive magnets are used as the nonlinear springs between gripper actuators and fingers. The position and force-stiffness characteristics of the fingers are adjusted simultaneously, by controlling the air-gaps between magnets. Finally, the application of the gripper in a nail hammering task is studied as an example of dynamic manipulation. For this purpose, an optimal stiffness control problem is solved to maximize the impact force of the hammering task through maximizing the kinetic energy of the grasped object at the hitting instance. Despite the simplicity of the design, experimental results indicate the effectiveness of the gripper for dynamic manipulation.
\end{abstract}

\section{INTRODUCTION}

In the past few years, there has been a growing interest in the development of Variable Stiffness Actuators (VSAs) as an alternative to conventional stiff joints for robot arms. Various mechanisms have been proposed to mechanically adjust the joint stiffness using compliant elements such as springs between the load and actuators [1]. Based on the task requirements, the stiffness of a VSA can be either increased, if the positioning accuracy is required, or decreased for a compliant interaction.

Generally, the inherent compliance and energy storage capability of VSAs offers two main advantages: (i) Contrary to stiff joints, the elastic elements of VSAs act as a low-pass filter by absorbing the impact energy and preventing serious damages to the robot/environment [2]. This characteristic can improve human safety in physical human-robot interactions; (ii) Task performance in fast and highly dynamic motions can be improved by exploiting the natural dynamics and energy storage capability of the elastic elements [3].

Although considerable research has been devoted to the design of VSAs in the form of rotary joints, yet less attention has been paid to the robot grippers with adjustable compliant actuation. In fact, the development of fully VSA-based arms with multiple degrees of freedom (DoF) requires an extra actuator at each joint which ultimately increases the complexity, size and the weight of the system [4]. Therefore, the design of variable stiffness grippers is a potential solution to achieve some of the key features of a fully VSA-based arm at a lower cost and complexity. By equipping conventional stiff arms with

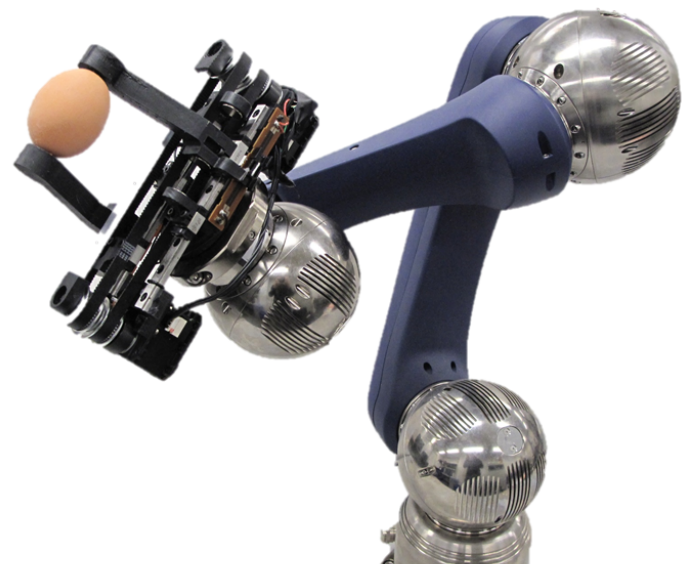

Fig. 1. The variable stiffness gripper with antagonistic magnetic springs.

variable stiffness grippers the dexterity, safety, and robustness of object manipulation can be enhances, particularly when the handling of fragile or potentially harmful objects is of interest (e.g., sharp tools or hot liquids).

In this regard, Zhang et al. [5] included a passive nonlinear compliant link into the design of a gripper to initially touch and detect target objects by estimating external forces as a function of link's length and then progressively adapt the gripper fingers to the shape of desired object based on the nonlinear stiffening of the compliant link. However, the link compliance in their design was not controllable and it only changed passively as a function of the link length.

Kim et al. [6] employed a VSA, which was primarily designed as a rotary joint, to develop a 1-DoF variable stiffness gripper. They demonstrated the efficacy of having an adjustable stiffness capability in the manipulation of fragile and heavy objects with compliant and stiff actuation, respectively. Although most of the previously proposed rotary VSA joints can be used as a potential actuator for a VSA-based gripper, a more specific design is required to decouple the passive motions of the fingers from each other. Structure-controlled variable stiffness [7] and soft grippers (e.g., [8, 9]) are other interesting approaches to modulate the grasping stiffness. However, the implementation of high bandwidth controllers, which is essential for dynamic an fast controlled movements, is challenging for these categories of gripper actuation.

In this paper, we first describe the mechanical design of a variable stiffness gripper with two parallel fingers as shown in Fig. 1] By decoupling passive motions of the fingers, external 
forces acting on each finger can be estimated separately. Furthermore, when an object is grasped, the simultaneous passive deflection of the fingers in the direction of external forces can be realized. This improves the safety and robustness of the grasp, particularly during collisions with stiff environments [10]. The nonlinear compliant actuation, which is essential for antagonistic VSAs, is generated by using permanent magnets in a repulsive configuration. Thanks to the non-contact force interaction between magnets, tolerance to misalignments and low frictional hysteresis are obtained.

In addition to the design aspects, this paper presents the application of the proposed gripper for dynamic manipulation. The term dynamic manipulation refers to the methods which exploit the natural dynamics of the grasped object, as a result of arm movements, to improve the task performance [11]. In other words, dynamic and quick motions of the robot arm give accelerations to the grasped object to perform tasks such as ball juggling [12], hammering [13] and re-grasping [14]. The energy storage capability, in addition to the controllable stiffness, empowers VSAs to realize dynamic tasks that require releasing a large amount of energy over a short period of time. In these applications, it is desired to achieve a maximum kinetic energy (at a specific time or position) by releasing the stored potential energy in the elastic elements.

Garabini et al. [13, 15] studied the optimal control principles of a 1-DoF rotary VSA during the execution of hitting tasks by maximizing the link velocity at a given final position/time. They showed that varying the stiffness during the execution of hitting tasks significantly improves the performance (final velocity of the link). Similarly, Braun et al. [16] utilized a 2-DoF VSA arm and applied optimal control theory to experimentally show the capability of VSAs to enhance ball throwing by maximizing the projectile's length.

In previous studies, VSAs are mostly designed and utilized as rotary joints between the arm links. However, performing dynamic manipulation with a variable stiffness gripper imposes extra constraints in terms of ensuring an appropriate grasp during arm motions. Furthermore, the dynamics of the grasped object is a function of not only the gripper actuators but also the end-effector motions. Thus, in this paper, we experimentally investigate the extent to which a variable stiffness gripper mounted on a conventional stiff arm can be used in dynamic manipulation. For this purpose, an optimal stiffness trajectory problem is formulated for a hammering task. The objective is to increase the impact force by maximizing the hammer's kinetic energy at the hitting instance of a wind-up motion. The results of numerical simulations and the hammering experiment are presented and discussed accordingly.

\section{Model of the Proposed GRIPPER}

\section{A. Design Concept}

Fig. 2 illustrates the concept of the proposed model for a parallel jaws/fingers gripper with variable stiffness actuation. In this model, each finger is driven by adjusting the endpoint positions of two nonlinear pre-loaded springs. Fingers

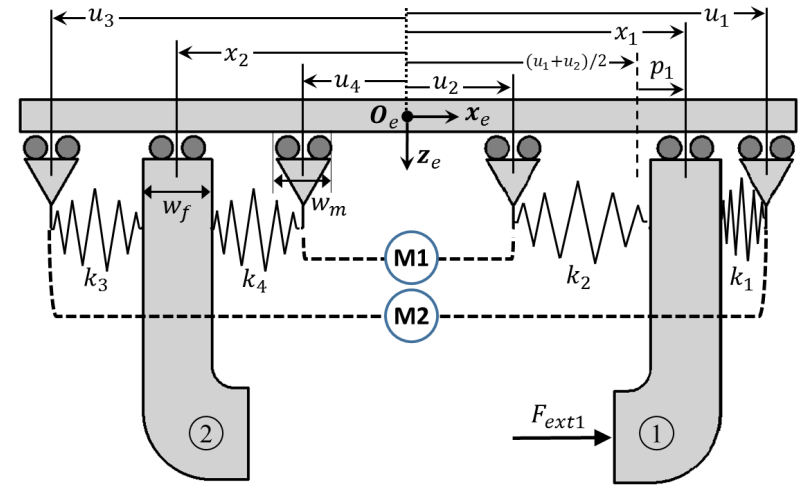

Fig. 2. Model of the proposed variable stiffness gripper with antagonistic pre-loaded nonlinear springs. M1 and M2 represent the mechanism's motors.

are mounted on passive (non-actuated) sliders whereas spring end-points are mounted on actuated sliders. Two antagonistic motors are enough to control the end-point positions of the springs by considering a symmetric magnet motion $\left(u_{3}=-u_{1}\right.$ and $u_{4}=-u_{2}$ in Fig. 2).

In a quasi-static approach, each finger tends to stay in the equilibrium position and altering the distance between the spring end-points leads to a change in force-deflection characteristics of the finger. In other words, translational motions of the fingers can be achieved by controlling the equilibrium position, whereas, the antagonistic nonlinear springs provide the capability of adjusting stiffness as a function control inputs $\left(u_{1}, u_{2}, u_{3}\right.$ and $\left.u_{4}\right)$. Therefore, the position control of spring end-points allows the stiffness control of the fingers as well as adjusting their position (before grasping) or contact force (after grasping) simultaneously. Linear position sensors (e.g., sliding potentiometers) are required to measure fingers' passive deflection from equilibrium $\left(x_{1,2}\right)$ and accordingly estimate external forces.

This design concept can provide the estimation of contact forces on each finger separately. Furthermore, it offers linear compliance along $x_{e}$ when an object is grasped and an external contact force exerts at the object/fingers. In this situation, the compliance of the gripper can protect the object and fingers from high impact forces as discussed in our previous work [10].

\section{B. Grasp Force and Stiffness Modulation}

With reference to Fig. 2 and neglecting the effect of gravity and friction, the static equilibrium of finger- 1 will be $\left(u_{1}+u_{2}\right) / 2$. Therefore, in the presence of external forces, the passive deflection of finger- 1 can be measured from its position feedback $x_{1}$ using (1),

$$
p_{1}=x_{1}-\left(u_{1}+u_{2}\right) / 2
$$

Since the springs are pre-loadeded, the following quasistatic relation holds,

$$
\sum F=F_{e x t 1}+f_{k 1}+f_{k 2}=0
$$

where $F_{\text {ext } 1}$ is the external force applying on finger- 1 and $f_{k i}$ denotes the repulsive force of $i^{\text {th }}$ magnetic spring with an 
end-point position of $u_{i}$ with a model which will be presented in (5). The directions of these forces and accordingly their signs in (2) can be determined based on the magnet locations with respect to the finger. Therefore, for a given deflection of $p_{1}$, the force and stiffness can be estimated by the following relations,

$$
\begin{gathered}
F_{\text {ext } 1}\left(p_{1}, u_{1}, u_{2}\right)=f_{k 1}-f_{k 2} \\
K_{1}\left(p_{1}, u_{1}, u_{2}\right)=\frac{d F_{e x t 1}}{d p_{1}}=\frac{d f_{k 1}}{d p_{1}}-\frac{d f_{k 2}}{d p_{1}}
\end{gathered}
$$

where $K_{1}$ is the finger stiffness as a function of its passive deflection and control inputs. Due to the antagonistic design, force and stiffness can be simultaneously modulated although they are not independent.

\section{Mechanical Design}

The overall assembly model and components of the developed gripper are illustrated in Fig. 3. This gripper is designed based on the proposed concept of Fig. 2 and permanent magnets in a repulsive configuration are used as the pre-loaded nonlinear springs. Magnetic springs are a good replacement for their mechanical counterparts by providing the system with compactness, lightness and low hysteresis effect [17]. Moreover, thanks to the non-contact force interactions between actuators and loads, magnetic springs offer precise force transmission and tolerance to misalignments. Such features led to a considerable improvement in the compliant behavior of the gripper in terms of reliability and robustness.

Miniature linear recirculating ball bearing sliders (Misumi, part no. SE2BSZ10) are used to provide low frictional and smooth translational motion of the actuated and passive (nonactuated) magnets. The fingers are mounted on the moving carts with passive magnets. Two sets of timing belt and pulleys are used to control the positions of the actuated magnets (pulleys with a pitch diameter of $25.4 \mathrm{~mm}$ and 40 teeth). The symmetric displacement of the fingers is achieved by attaching

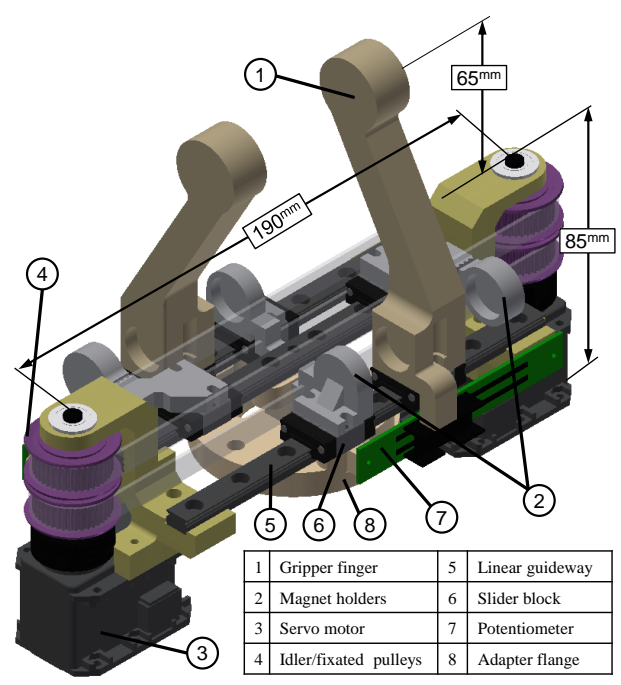

Fig. 3. The assembly model of the gripper. Belts are made transparent in the image to enhance the model comprehensibility. the outer actuated magnets to both sides of a belt and similarly the inner actuated magnets to the other belt. The gripper's size and geometry are optimized based on the robot arm of Fig. 1 .

If both of the fingers are placed on a single linear rail then there will be a motion constraint imposed by the slider motions due to their widths $\left(u_{2}-u_{4} \geq w_{m}\right)$. This constraint will affect the feasible force-stiffness bandwidth of the fingers, particularly for small objects with small distances between fingers, as discussed in [10]. We have addressed this limitation by mounting finger sliders on two separate parallel rails and the resultant offset between them are taken into account by an oblique design of the fingers (see Fig. 3). Furthermore, a third linear rail is used between the two finger rails to increase the gripper stroke since the widths of the magnets are smaller than sliders.

Two identical servo motors (Dynamixel RX-24F) in position control mode with a resolution of $0.29^{\circ}$ are used to drive the actuated magnets. This servo motor can provide a relatively high speed which is essential for dynamic manipulation. Note that two pulleys are mounted on each motor axis. One is fixated on the shaft to transfer motor torque and the other one is just an idler to maintain tension in the belt. The displacements of the actuated magnets $\left(u_{1 . .4}\right)$ are indirectly measured via the angular positions of the servos and the fingers' positions $\left(x_{1,2}\right)$ are directly measured using sliding potentiometer sensors. The analog outputs of these sensors are converted to digital signals with 10-bit resolution for the experiments.

Magnets are chosen as identical cylindrical neodymium magnets with a diameter and thickness of 15 and $6 \mathrm{~mm}$, respectively. The magnetization strength of these magnets according to the manufacturer's datasheet is approximately $6.05 \times 10^{5}(\mathrm{~A} / \mathrm{m})$. The size and flux density of the magnets can be selected based on the stiffness-force requirements. This requires the modeling of magnet repulsion force with respect to the air-gap which is discussed in the next section.

\section{Magnetic Repulsion Force}

Analytical modeling of the attractive and repulsive forces between magnets is not a trivial procedure because of their nonlinearities and dependency to various parameters such as geometry, orientation, flux density, and air-gap. A mathematical model to estimate the magnetic repulsion between two cylindrical magnets is presented in [18] by assuming magnets as electric dipoles. To check the validity of the electric dipoles model, repulsion forces versus air-gaps are measured experimentally between a pair of magnets used in the gripper mechanism. Figure 4 shows the experimental results and the analytical curve for the gripper's magnets. The significant difference between the actual data points and analytical model especially for small air-gaps $(s / h<2)$ is mainly due to the assumption of electric dipoles. This makes the electric dipoles model unsuitable for the case of our gripper since the desired range of air-gap is usually not large for this application.

Figure 4 also depicts the results of Finite Element Method which are obtained from FEMM software [19]. Numerical 

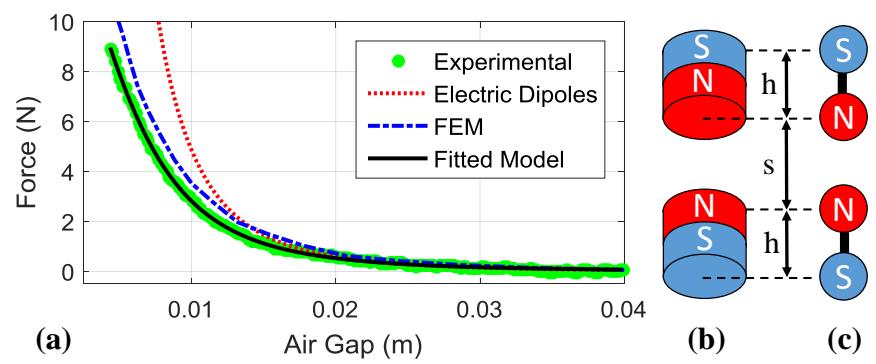

Fig. 4. (a) Fitted model for magnetic repulsion force with the identified parameters of $a=4.9 \mathrm{e}-6, b=5.3 \mathrm{e}-5$ and $c=2.3 \mathrm{e}-7$; (b) and (c) show the model of actual magnets and electric dipoles, respectively.

results of FEM analysis are close to the actual measurements. However, there is a consistent positive difference (FEMactual $>0$ ) which is due to the non-ideal magnetic field of actual magnets. This indicates that FEM results are promising for design purposes, however, an analytical model is essential particularly for control purposes.

To obtain a mathematical model for the repulsion force, a modified inverse power law expressed in (5) is used for fitting to the experimental data. This model is presented in relation (5) which is selected according to the previous studies [10, 20].

$$
f_{k}(s)=\frac{a}{s^{3}+b s+c}
$$

$a, b$ and $c$ are the model parameters and $s$ is the air-gap between the magnets. Matlab nonlinear least-squares solver "Isqnonlin" with trust-region method is used to estimate the model parameters. The result of model fitting is shown in Fig. 4 and used as spring forces in the gripper model $\left(f_{k i}\right)$. In this paper, the analytical approximation of the magnet repulsion (5) is used in the formulations and simulation of hammering with dynamic manipulation which is presented in the next section. However, this model can also be used to adjust the desired force-stiffness characteristic during grasp operations as formulated in (3) and (4).

\section{Hammering With Dynamic Manipulation}

In addition to improving grasp safety and robustness through adjusting fingers' passive compliance, the variable stiffness fingers can be used to enhance the performance of a class of dynamic manipulation tasks in which a high object velocity larger than the end-effector velocity is required in a short period of time. The controllable grasp stiffness allows the system to store potential energy and release it over a short time before reaching the target point. One common dynamic manipulation task is hitting and the optimal arm trajectory for hitting scenarios are usually a back and forth (wind-up) motion before reaching the impact point [13, 15, 16].

As a case study, we show how the adjustable stiffness can be used to increase the impact force of a hammering task at the hitting point of a wind-up arm motion (see Fig. 5). This can be achieved by solving a Trajectory Optimization Problem (TOP) that seeks to find an optimal stiffness trajectory that minimizes a desired cost function and satisfies a set of constraints. For the case of hammering, the objective is to increase the impact

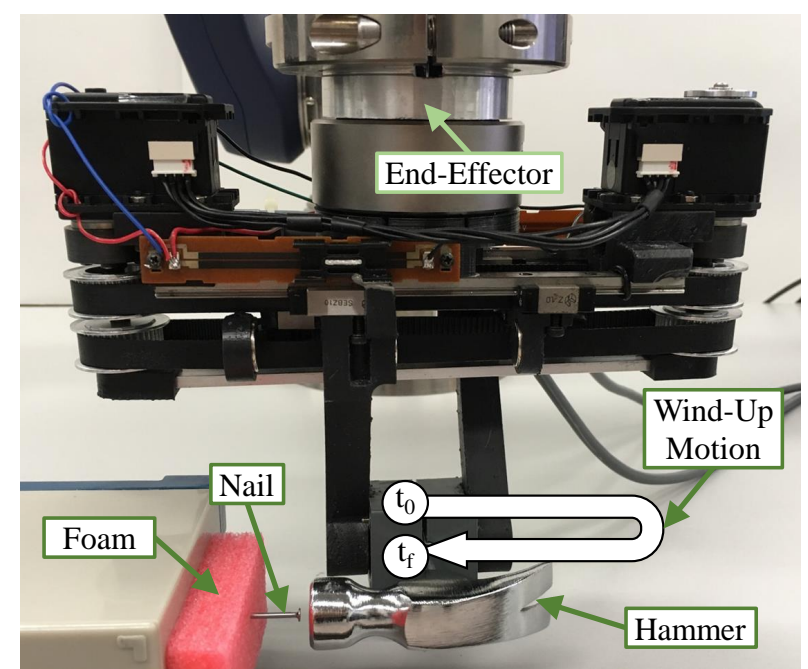

Fig. 5. Experimental setup for hammering with dynamic manipulation.

force by maximizing the hammer's kinetic energy at the hitting point (the nail's head position).

\section{A. Problem Formulation}

Representation of the cost function for our hammering task is quite straightforward and only depends on the kinematic aspects of the grasped object (the hammer) at the hitting point (the nail's head). According to Fig. 2 and 7, to maximize the hammer velocity at a terminal position of $x_{f}$, TOP can be formulated in the framework of optimal control as below,

$$
\min _{u_{1,2}} J=-\dot{x}_{o}^{e}\left(x_{f}\right)
$$

subject to the object dynamics

$$
\dot{\mathbf{x}}(t)=\mathbf{f}(\mathbf{x}(t), \mathbf{u}(t), t)
$$

and constraints

$$
\left\{\begin{array}{l}
w_{o} / 2+w_{m} / 2+w_{f} \leq u_{1}(t) \leq u_{1 \max } \\
u_{2 \min } \leq u_{2}(t) \leq w_{o} / 2-w_{m} / 2 \\
\left|\dot{u}_{1,2}\right| \leq \dot{u}_{\max } \\
f_{k 1}>f_{k 2} \text { and } f_{k 3}>f_{k 4}
\end{array}\right.
$$

where $J$ is the performance criterion; $\mathbf{x}(t)$ is the state vector; $\dot{x}_{o}^{e}$ is the velocity of the hammer with respect to end-effector frame; $w_{f}, w_{m}$ and $w_{o}$ denote the width of the fingers, actuated magnets and grasped object, respectively (these geometries are shown in Fig. 2p.

Regarding the problem constraints presented in (8), the first two ones are mainly imposed by the motion limitations of the mechanism; the third one is the maximum motor speed and the last constraint ensures an active contact between the fingers and the grasped hammer. The numerical values for the constraints of our case study are listed in Table I]

The equality constraint of (7) represents the hammer dynamics which will be presented in section [III-C Note that in the presented formulation, for the sake of simplicity, the trajectory of the arm joints has been considered to be predefined as 
TABLE I

PARAMETER VALUES OF THE TOP CONSTRAINTS.

\begin{tabular}{lcccccc}
\hline Parameter & $w_{f}$ & $w_{m}$ & $w_{o}$ & $u_{1 \max }$ & $u_{2 \min }$ & $\dot{u}_{\max }$ \\
\hline Value & $21 \mathrm{~mm}$ & $5 \mathrm{~mm}$ & $35 \mathrm{~mm}$ & $60 \mathrm{~mm}$ & $-30 \mathrm{~mm}$ & $0.15 \mathrm{~m} / \mathrm{s}$ \\
\hline
\end{tabular}

described in the next section. Thus, the optimization problem will be solved only for the gripper actuators $\left(u_{1}, u_{2}\right)$. For a more general hitting scenario with moving targets, for instance ball juggling, one may consider the arm joints or the endeffector pose as a design parameter as well and include their constraints in the problem formulation.

\section{B. End-Effector Trajectory}

A predefined 1-DoF end-effector trajectory is used to simplify grasped hammer dynamics and solve the TOP only for the gripper actuators. To obtain a straight back and forth endeffector motion similar to common hitting tasks and previous studies [13, 15], a modified version of skew-normal density function $(9)$ has been used in the Cartesian space. Thanks to the skewness factor $(\alpha)$, an asymmetric trajectory with a larger velocity in the forward than backward motion is obtained that is similar to what humans do during hammering. This helps to the robustness of the grasping in the sudden change of velocity direction at the switching point of the wind-up motion by limiting the kinetic energy of the hammer before this point.

$$
x_{e}^{b}(t)=A \phi\left(t^{\prime}\right) \Phi\left(\alpha t^{\prime}\right)
$$

where $A$ is used to adjust the amplitude of the motion, and

$$
\begin{aligned}
& \phi(t)=\frac{1}{\sqrt{2 \pi}} e^{-t^{2} / 2} \\
& \Phi(t)=\frac{1}{2}\left(1+\operatorname{erf}\left(\frac{t}{\sqrt{2}}\right)\right) \\
& t^{\prime}=t_{1}+\frac{t_{2}-t_{1}}{T} t
\end{aligned}
$$

where $\phi(t)$ denotes the standard normal density function with the cumulative function of $\Phi(t)$; erf is the error function; $t^{\prime}$ is defined to map the original skew-normal function to the desired time interval $[0, T] ; t_{1}$ and $t_{2}$ denote the desired starting and ending interval of the original skew-normal function, respectively.

Figure 6 depicts the resulting displacement, velocity and acceleration profile for the parameter values of $\alpha=-2.5$, $A=-0.12, T=0.55 s, t_{1}=-3.1$ and $t_{2}=0.9$ which will be used for numerical solution and implementation of the hammering task. The orientation of the end-effector within this motion is kept constant such that the gripper moves only horizontally along the $x_{e}$ direction. This straight motion in the Cartesian space significantly simplifies the hammer dynamics (18) during arm motions which will be discussed in the next section. The trajectories of the arm joints for the given Cartesian motion are obtained from the inverse kinematics relation of the arm.

Note that $A, T$, and $\alpha$ values for the chosen trajectory are tuned experimentally such that for a constant low-stiffness
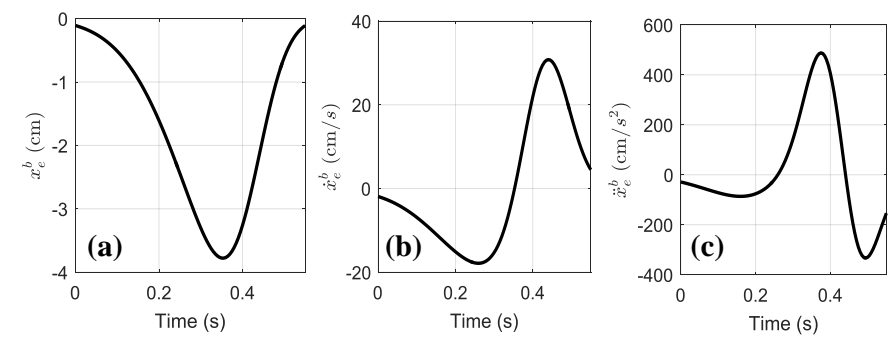

Fig. 6. The back and forth motion of the end-effector for hammering experiment in terms of end-effector (a) displacement, (b) velocity and (c) acceleration.

setup a relatively high hammering velocity at the terminal position can be achieved. This constant low-stiffness setup is used for comparison purposes with the solution of the TOP to evaluate performance improvement via an optimal stiffness trajectory.

\section{Grasped Object Dynamics}

In the formulated TOP, the equality constraint (7) represents the hammer dynamics imposed by the arm motions and gripper actuators. Thus, an analytical expression of the grasped object dynamics must be derived before solving the TOP. Figure 7 illustrates a planar grasp with the variable stiffness gripper which is mounted on a robotic arm with $n$ (rotational) joints and a generalized coordinates of $\mathbf{q} \in \mathbf{R}^{n}$. Two frames are defined including the base $O_{b}-x_{b} y_{b} z_{b}$ and end-effector frame $O_{e}-x_{e} y_{e} z_{e}$. The hammer dynamics in (7) can be rewritten into state-space representation for the TOP, with $\mathbf{x}_{1}$ and $\mathbf{x}_{2}$ as the object position and velocity with respect to the end-effector frame $O_{e}$, respectively.

$$
\begin{cases}\dot{\mathbf{x}}_{1} & =\mathbf{x}_{2}=\dot{\mathbf{p}}_{o}^{e} \\ \dot{\mathbf{x}}_{2} & =\ddot{\mathbf{p}}_{o}^{e}\end{cases}
$$

In general, the absolute translational velocity of the object

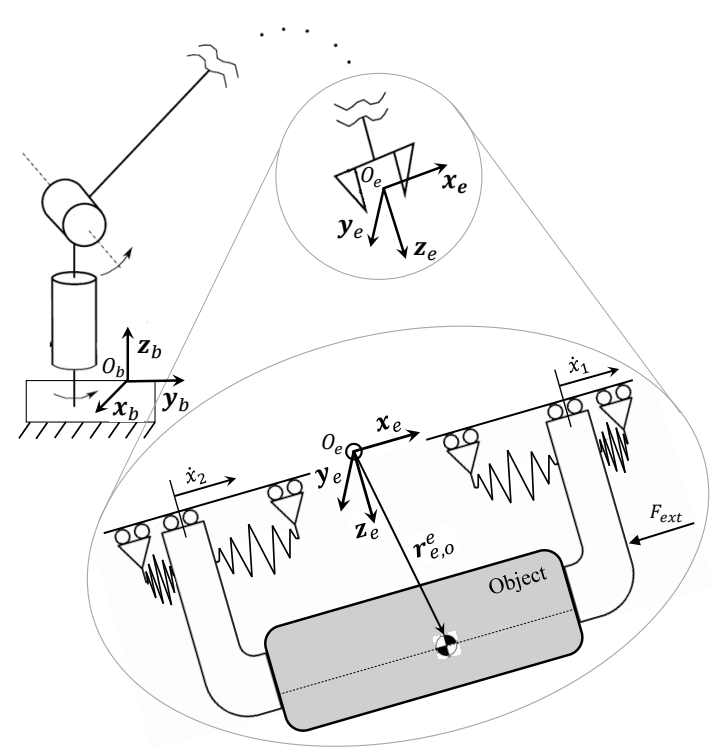

Fig. 7. Schematic of a planar grasp using the variable stiffness gripper 
with respect to the end-effector frame can be written as (14),

$$
\dot{\mathbf{p}}_{o}^{e}=\mathbf{R}^{T} \dot{\mathbf{p}}_{o}^{b}=\mathbf{R}^{T}\left(\mathbf{J}_{p} \dot{\mathbf{q}}+\mathbf{S}\left(\boldsymbol{\omega}_{e}^{b}\right) \mathbf{r}_{e, o}^{b}+\mathbf{R}\left[\dot{x}_{1}, 0,0\right]^{T}\right)
$$

where $\mathbf{R}$ is the rotation matrix of frame $O_{e}$ with respect to $O_{b} ; \mathbf{J}_{p}$ is the $(3 \times n)$ Jacobian matrix associated with endeffector linear velocity; $\boldsymbol{\omega}_{e}^{b}$ is the absolute angular velocity of the gripper expressed in the base frame; $\mathbf{S}($.$) denotes the skew-$ symmetric matrix operator and $\dot{x}_{1}$ is the relative speed of the object with respect to the end-effector frame (note that $\dot{x}_{1}=$ $\dot{x}_{2}$ since a firm grasp is assumed). Furthermore, according to the relationship between angular velocity and rotation matrix [21], the following relation holds,

$$
\mathbf{R S}\left(\boldsymbol{\omega}_{e}^{b}\right) \mathbf{R}^{T}=\mathbf{S}\left(\mathbf{R} \boldsymbol{\omega}_{e}^{b}\right)=\mathbf{S}\left(\boldsymbol{\omega}_{e}^{e}\right)
$$

Thus, (14) can be rewritten as (16,

$$
\dot{\mathbf{p}}_{o}^{e}=\mathbf{R}^{T} \mathbf{J}_{p} \dot{\mathbf{q}}+\mathbf{S}\left(\boldsymbol{\omega}_{e}^{e}\right) \mathbf{r}_{e, o}^{e}+\left[\dot{x_{1}}, 0,0\right]^{T}
$$

where $\boldsymbol{\omega}_{e}^{e}$ is the absolute angular velocity of the gripper in $O_{e}$ frame. The translational Newton-Euler formulation can be expressed in the end-effector frame as below,

$$
m\left(\ddot{\mathbf{p}}_{o}^{e}+\mathbf{S}\left(\boldsymbol{\omega}_{e}^{e}\right) \dot{\mathbf{p}}_{o}^{e}-\mathbf{R}^{T} \mathbf{g}\right)=\mathbf{f}^{e}
$$

where $\ddot{\mathbf{p}}_{o}^{e}$ is the absolute translational acceleration of the object with respect to the end-effector frame $\left(\mathrm{O}_{e}\right) ; \mathrm{m}$ is the combined mass of the object and fingers; $g$ is the gravity vector; $\mathbf{f}^{e}=\left[f_{x}^{e}, f_{y}^{e}, f_{z}^{e}\right]^{T}$ is the input force vector. However, given the straight horizontal end-effector trajectory of (9), the effect of gravity is eliminated and the angular velocity of the hammer will be zero. Thus, (16) and (17) can be simplified and the state-space representation of the hammer dynamics can be written as below,

$$
\left\{\begin{array}{l}
\dot{\mathbf{x}}_{1}=\mathbf{x}_{2}=\mathbf{R}^{T} \mathbf{J}_{p} \dot{\mathbf{q}}+\left[\dot{\left.x_{1}, 0,0\right]^{T}}\right. \\
\dot{\mathbf{x}}_{2}=\ddot{\mathbf{p}}_{o}^{e}=\frac{1}{m} \mathbf{f}^{e}
\end{array}\right.
$$

Note that for our TOP, the equation of motion is only needed to be considered in $x_{e}$ coordinate, since it is along the compliant motions of the fingers and the hammering task. Thus, for the right side of the equation of motion [18) in $x_{e}$ coordinate we have

$$
f_{x}^{e}=\sum_{i=1}^{4} f_{k i}+F_{v} \dot{x}_{1}+F_{s} \operatorname{sgn}\left(\dot{x}_{1}\right)+F_{\text {ext }}
$$

where $f_{k i}$ is the force exerted from the magnetic spring $i$; $F_{e x t}$ is the projection of external forces along $x_{e}$ axis; $F_{v}$ and $F_{s}$ are viscous and Coulomb friction coefficients, respectively. Due to the low static friction of the recirculating balls of the sliders, the Coulomb friction was found to be very small and thus it was neglected. However, a viscus coefficient of $F_{v}=0.45 \mathrm{Ns} / \mathrm{m}$ was identified based on the decay rate of free oscillations of a finger after releasing it from an arbitrary initial deviations.

\section{Numerical Solution}

Given the end-effector motions, we aim to solve the TOP (6) for the gripper actuators $u_{1,2}(t)$. To solve TOPs, based on the level of complexity, analytical or numerical solutions may be used. In general, there are three numerical methods for solving TOPs numerically including dynamic programming, indirect, and direct methods. Garabini et al. [15] investigated the optimal control problem of VSAs in a kicking task using a direct method by discretization and multiple shootings method. Barun et al. [16] utilized an iterative linear quadratic regulator (iLQR) algorithm to solve an optimal control problem with an objective to maximize ball throwing distance with a 2DoF VSA arm. Zhakatayev et al. [22] used a nonlinear model predictive controller to track generated reference trajectories of an optimal control problem.

In this study, the non-linearity of the hammer dynamics and constrained range of magnet displacements impose significant challenges to solve the associated TOP analytically. Therefore, a numerical direct method is utilized to transcribe the TOP to a nonlinear programming problem (NLP) [23]. The transcription is done by parameterizing the unknown trajectories as Bsplines and then rewriting the optimization problem in terms of the B-spline parameters. Our goal is to verify the effectiveness of the proposed approach to predict intuitive, but not trivial, solutions before utilizing it in more complex tasks where endeffector motion is included as a design trajectory as well.

A Spline is a curve composed of a set of polynomials which are connected at their break-points, satisfying a given degree of smoothness. Splines can be represented using Bspline functions in a computationally efficient form. A Bspline is defined as

$$
f(t)=\sum_{k=1}^{N_{c}} \alpha_{k} B_{k, r}(t)
$$

where $\alpha_{k}$ is the $\mathrm{k}^{t h}$ free parameter of the $\mathrm{B}$-spline; $B_{k, r}(t)$ is the spline basis function; $N_{c}$ is the number of free parameters which equals to $N_{c}=N \times(r-s)+s ; N$ denotes the number of polynomial pieces constructing the spline; $r$ is the order of polynomial pieces and $s$ is the smoothness condition at the break points ( $r$ must be at least equal to $s$ ). Since the computation of the derivative of a B-spline is straightforward, the derivative constraints can be easily computed. The parameters $N, r$, and $s$ should be tuned such that a sufficient degree of freedom for a given trajectory is ensured. In our TOP, three main problem trajectories including the hammer's displacement $\left(\mathbf{p}_{o}^{e}\right)$ and th actuated magnets' displacement $\left(u_{1,2}\right)$ are parametrized using the B-spline function 20 . The associated parameters of these trajectories used in this study are listed in Table II

TABLE II

PARAMETRIZED TRAJECTORIES WITH B-SPLINES.

\begin{tabular}{cccc}
\hline \hline Trajectory & Pieces $(N)$ & Order $(r)$ & Smoothness $(s)$ \\
\hline $\mathbf{p}_{o}^{e}$ & 10 & 5 & 4 \\
\hline$u_{1}$ & 4 & 3 & 2 \\
\hline$u_{2}$ & 4 & 3 & 2 \\
\hline
\end{tabular}

By parameterizing the unknown trajectories using B-splines, 


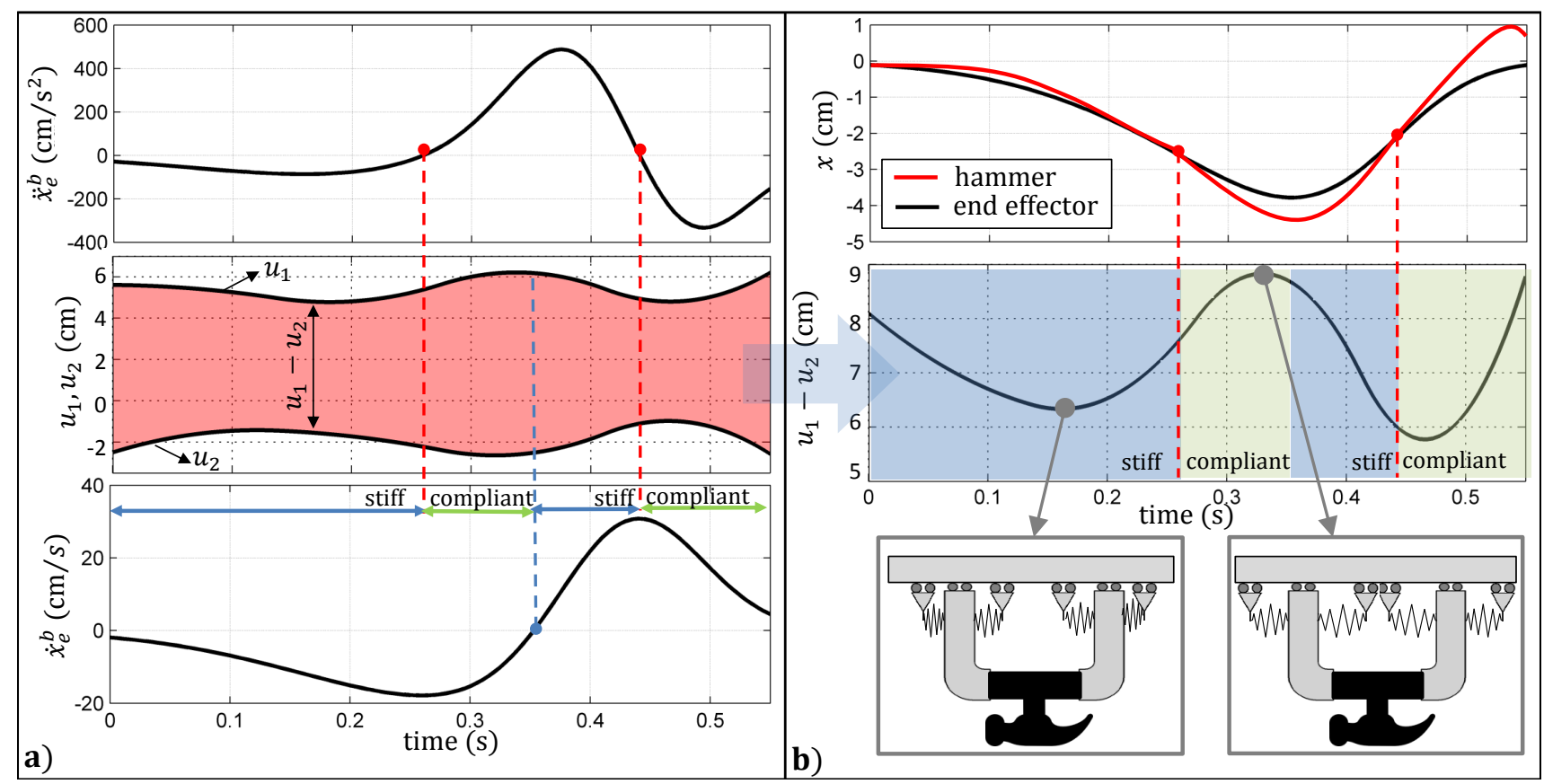

Fig. 8. a) The optimal trajectory of the actuated magnets $\left(u_{1}, u_{2}\right)$ in the middle plot is compared to the desired velocity $\left(\dot{x}_{e}^{b}\right)$ and acceleration of the end-effector $\left(\ddot{x}_{e}^{b}\right)$ to define stiff and compliant region. b) hammer versus end-effector position. $\left(u_{1}-u_{2}\right)$ is inversely related to the stiffness.

the transcription approach transforms the TOP expressed by (6), (7), and (8) into a NLP as below,

$$
\min _{\mathbf{z} \in \mathbf{R}^{N_{c}}} F(\mathbf{z})
$$

subject to

$$
l b \leq\left(\begin{array}{c}
\mathbf{z} \\
A \mathbf{z} \\
G(\mathbf{z})
\end{array}\right) \leq u b
$$

where $\mathbf{z}$ is the vector of $\mathbf{B}$-spline free parameters $\left(\alpha_{1}, \ldots, \alpha_{N_{c}}\right)$; $A$ is the matrix representing linear constraints; $G(z)$ denotes nonlinear constraints which in this case includes the hammer dynamics. As the TOP converted to an NLP, the cost function and constraints are evaluated and enforced at collocation points which are generally denser than the breakpoints. In this study, a total number of 25 equally spaced collocation points are defined within the task time interval $[0, T]$. This transcription method may lead to a suboptimal solution since optimality is determined in the subspace of B-splines defined by $N, r$, and $s$. Thus, changing these parameters may affect the suboptimal solution.

The transcription was done by OPTRAGEN toolbox [24] and Galerkin projection method is used to impose the nonlinear dynamics $(18)$ as an equality constraint. To solve the transcribed optimization problem, SNOPT software package [25] was used, since the resulting NLP had a large number of sparse linear constraints. To enhance the numerical solution of such problems, SNOPT employs a sequential quadratic programming method with limited-memory quasi-Newton approximations to the Hessian of Lagrangian.

The obtained solutions for the optimal displacements of the actuated magnets $\left(u_{1}\right.$ and $\left.u_{2}\right)$ are shown in Fig. 8 . With reference to Fig. 2 when an object is grasped, by decreasing $u_{1}$, the air-gaps between the outer actuated magnets and the fingers will be decreased resulting in a larger grasp force and higher stiffness. In terms of inner magnets, however, increasing $u_{2}$ leads to a smaller air-gap between the inner actuated magnets and the fingers and ultimately a lower grasp force. From the obtained optimal trajectories and end-effector motions, it can be interpreted that stiffness starts increasing as the backward speed of the end-effector increases. After reaching the maximum speed, the stiffness decreases such that a maximum potential energy can be stored in the magnetic springs at the point where the direction of the movement changes. Again by increasing the forward speed, the stiffness increases to force the hammer move faster along the endeffector direction of motion. Finally, after reaching the maximum forward speed, the stiffness reduces to let the hammer continues moving even after the deceleration and stopping the end-effector.

\section{IMPLEMENTATION}

Figure 5 depicts the experimental setup consisted of the variable stiffness gripper mounted on the end-effector of a 6-DoF lightweight robot arm (SCHUNK PowerBall LWA [26]). A small hammer is used to hammer a nail into a foam pad with a rigid base. The end-effector tracked the straight back and forth trajectory presented in Fig. 6 while the gripper servo motors were used to control the positions of the actuated magnets $\left(u_{1}\right.$ and $\left.u_{2}\right)$. Three trials with different grasp stiffnesses were conducted: (i) a constant high-stiffness setup, (ii) a constant low-stiff setup that was previously used in Section III-B for the design of the end-effector trajectory, and 

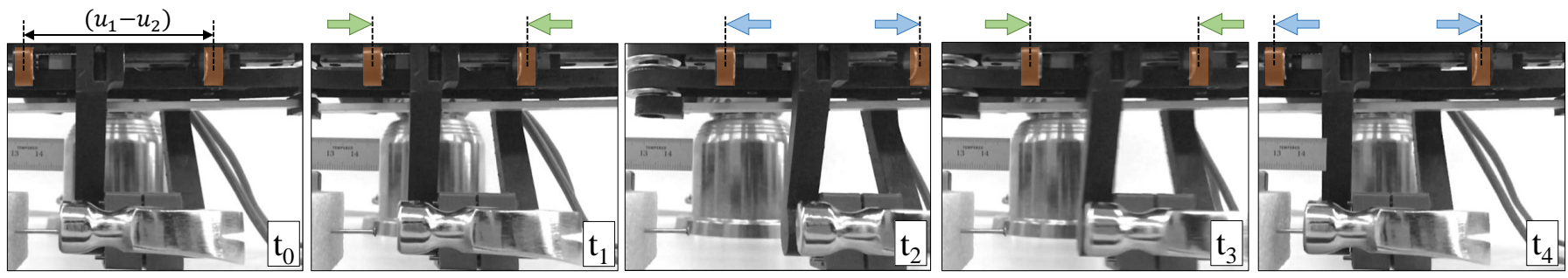

Fig. 9. Trial sequence of the hammering task with variable stiffness actuation. Actuated magnets are marked with the transparent rectangles. Green arrows denote increased stiffness whereas the blue ones correspond decreased stiffness. Experimental video: https://youtu.be/-QA6ptfptes

(iii) a variable stiffness associated with the optimal stiffness which was numerically obtained by solving the TOP (6). An image sequence of the hammering task with the optimal variable stiffness is shown in Figure 9

The hammer displacement with respect to the base frame is shown in Fig. 10 for the three cases of stiffness setup. From the experimental measurements, it can be observed that the maximum penetration of the nail into the foam for the variable stiffness is $2 \mathrm{~mm}$ more than the constant low stiffness. Note that the position of the nail is located at $-1 \mathrm{~mm}$ in $\mathrm{X}$-coordinate. According to the results, it can be concluded that using an adjustable grasping stiffness the performance of dynamic manipulation tasks such as hitting can be improved.

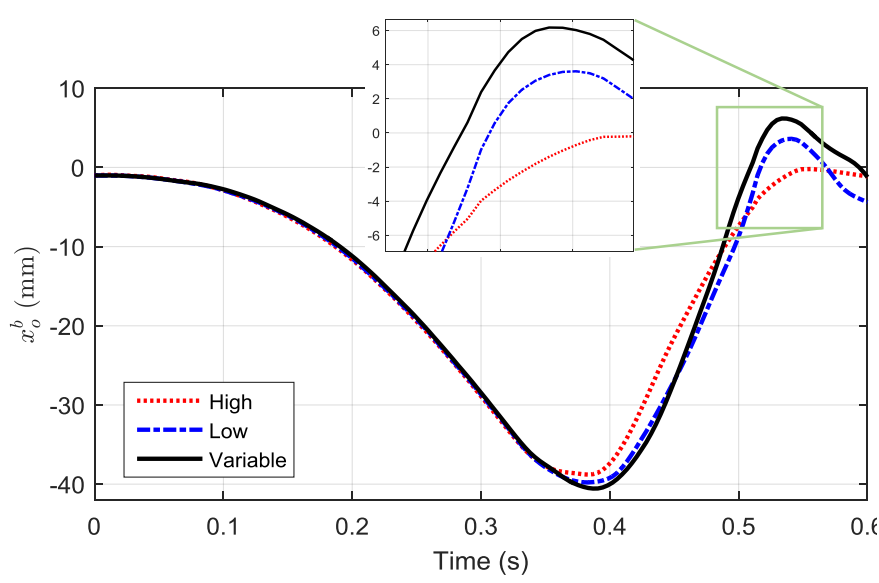

Fig. 10. Experimental results for the 3 cases of stiffness setups. The graph shows the displacement of the hammer with respect to the base frame in Xcoordinate. The nail is located at $-1 \mathrm{~mm}$ and the TOP seeks to maximize the compliance displacement of the hammer at the hitting instance by maximizing its velocity at this point.

There were some limitations for the proposed gripper in performing dynamic manipulation tasks. First, it should be noted that the improvement in the objective function highly depends on the dynamic features of the actuators. For instance, high bandwidth gripper motors and fast magnet positioning can better improve the performance of the task. Second, the feasible range of stiffness during manipulation is affected by the minimum required grasping force since the force and stiffness modulation are not independent (as discussed in section II.B). A large grasping force is required if we only rely on the friction between the finger-tips and the grasped objects that limits the feasible range for low stiffness setups.
To address this issue, high frictional materials at the fingertips or mechanically engaged shapes can be used for grasping.

\section{CONClusion}

This article presented a two-finger variable stiffness gripper and its application to dynamic manipulation. In the design of gripper, antagonistic permanent magnets are used as nonlinear springs to modulate position and force-stiffness characteristics of the fingers, simultaneously. By measuring the finger displacements, contact forces acting on each of the fingers can be estimated independently. Furthermore, mechanism compliance can improve the safety of grasp if an external force exerts at the grasped object/fingers.

The application of the proposed gripper is discussed for dynamic manipulation, where the controllable stiffness is exploited to improve the performance of a hammering task. The optimal trajectories of the gripper actuators were obtained numerically based on an optimal control framework and the results are validated experimentally. The results demonstrate the effectiveness of the gripper with adjustable stiffness for hitting tasks. Therefore, by equipping conventional stiff arms with the proposed variable stiffness gripper, some of the key features of a fully VSA-based arm can be realized at a lower cost and complexity.

In the future, we intend to extend our design by considering more fingers and higher degrees of freedom (planar compliance [27]). Furthermore, the application of the adjustable grasping stiffness in robot handover of objects to human is another interesting topic that can be investigated [28].

\section{REFERENCES}

[1] B. Vanderborght, A. Albu-Schaeffer, A. Bicchi, E. Burdet, D. Caldwell, R. Carloni, M. Catalano, O. Eiberger, W. Friedl, G. Ganesh, M. Garabini, M. Grebenstein, G. Grioli, S. Haddadin, H. Hoppner, A. Jafari, M. Laffranchi, D. Lefeber, F. Petit, S. Stramigioli, N. Tsagarakis, M. Van Damme, R. Van Ham, L. Visser, and S. Wolf, "Variable impedance actuators: A review," Robotics and Autonomous Systems, vol. 61, no. 12, pp. 1601-1614, dec 2013.

[2] S. Wolf, G. Grioli, O. Eiberger, W. Friedl, M. Grebenstein, H. Hppner, E. Burdet, D. G. Caldwell, R. Carloni, M. G. Catalano, D. Lefeber, S. Stramigioli, N. Tsagarakis, M. V. Damme, R. V. Ham, B. Vanderborght, L. C. Visser, A. Bicchi, and A. Albu-Schffer, 
"Variable stiffness actuators: Review on design and components," IEEE/ASME Transactions on Mechatronics, vol. 21, no. 5, pp. 2418-2430, Oct 2016.

[3] J. Nakanishi and S. Vijayakumar, "Exploiting passive dynamics with variable stiffness actuation in robot brachiation," Robotics: Science and Systems VIII, p. 305, 2013.

[4] N. G. Tsagarakis, I. Sardellitti, and D. G. Caldwell, "A new variable stiffness actuator (compact-vsa): Design and modelling," in 2011 IEEE/RSJ International Conference on Intelligent Robots and Systems, Sept 2011, pp. 378 383.

[5] Z. Zhang, A. Rodriguez, and M. T. Mason, "A novel nonlinear compliant link on simple grippers," in Intelligent Robots and Systems (IROS), 2015 IEEE/RSJ International Conference on. IEEE, 2015, pp. 29232928.

[6] B.-S. Kim and J.-B. Song, "Object grasping using a 1 DOF variable stiffness gripper actuated by a hybrid variable stiffness actuator,' in 2011 IEEE International Conference on Robotics and Automation. IEEE, may 2011, pp. 4620-4625.

[7] X. Li, W. Chen, W. Lin, and K. H. Low, "A variable stiffness robotic gripper based on structure-controlled principle," IEEE Transactions on Automation Science and Engineering, 2017.

[8] A. Firouzeh and J. Paik, "Grasp mode and compliance control of an underactuated origami gripper using adjustable stiffness joints," IEEE/ASME Transactions on Mechatronics, vol. 22, no. 5, pp. 2165-2173, Oct 2017.

[9] R. Adachi, Y. Fujihira, and T. Watanabe, "Identification of danger state for grasping delicate tofu with fingertips containing viscoelastic fluid," in Intelligent Robots and Systems (IROS), 2015 IEEE/RSJ International Conference on. IEEE, 2015, pp. 497-503.

[10] A. H. Memar, N. Mastronarde, and E. T. Esfahani, "Design of a novel variable stiffness gripper using permanent magnets," in Robotics and Automation (ICRA), 2017 IEEE International Conference on. IEEE, 2017, pp. 2818-2823.

[11] N. Furukawa, A. Namiki, S. Taku, and M. Ishikawa, "Dynamic regrasping using a high-speed multifingered hand and a high-speed vision system," in Proceedings 2006 IEEE International Conference on Robotics and Automation, 2006. ICRA 2006., May 2006, pp. 181-187.

[12] D. Serra, F. Ruggiero, V. Lippiello, and B. Siciliano, "A nonlinear least squares approach for nonprehensile dualhand robotic ball juggling," IFAC-PapersOnLine, vol. 50, no. 1, pp. 11 485-11490, 2017.

[13] M. Garabini, A. Passaglia, F. Belo, P. Salaris, and A. Bicchi, "Optimality principles in variable stiffness control: The vsa hammer," in Intelligent Robots and Systems (IROS), 2011 IEEE/RSJ International Conference on. IEEE, 2011, pp. 3770-3775.

[14] N. C. Dafle, A. Rodriguez, R. Paolini, B. Tang, S. S. Srinivasa, M. Erdmann, M. T. Mason, I. Lundberg, H. Staab, and T. Fuhlbrigge, "Extrinsic dexterity: In- hand manipulation with external forces," in 2014 IEEE International Conference on Robotics and Automation (ICRA), May 2014, pp. 1578-1585.

[15] M. Garabini, A. Passaglia, F. Belo, P. Salaris, and A. Bicchi, "Optimality principles in stiffness control: The vsa kick," in Robotics and Automation (ICRA), 2012 IEEE International Conference on. IEEE, 2012, pp. 33413346.

[16] D. J. Braun, M. Howard, and S. Vijayakumar, "Exploiting variable stiffness in explosive movement tasks," Robotics: Science and Systems VII, p. 25, 2012.

[17] A. Sudano, D. Accoto, L. Zollo, and E. Guglielmelli, "Design, development and scaling analysis of a variable stiffness magnetic torsion spring," International Journal of Advanced Robotic Systems, vol. 10, 2013.

[18] D. Vokoun, M. Beleggia, L. Heller, and P. Šittner, "Magnetostatic interactions and forces between cylindrical permanent magnets," Journal of magnetism and Magnetic Materials, vol. 321, no. 22, pp. 3758-3763, 2009.

[19] D. Meeker, "Finite element method magnetics," FEMM, vol. 4, p. 32, 2010.

[20] P. Onorato, P. Mascheretti, and A. DeAmbrosis, "Investigating the magnetic interaction with geomag and tracker video analysis: static equilibrium and anharmonic dynamics," European Journal of Physics, vol. 33, no. 2, p. 385, 2012.

[21] B. Siciliano, L. Sciavicco, L. Villani, and G. Oriolo, Robotics: modelling, planning and control. Springer Science \& Business Media, 2010.

[22] A. Zhakatayev, M. Rubagotti, and H. A. Varol, "Closedloop control of variable stiffness actuated robots via nonlinear model predictive control," IEEE Access, vol. 3, pp. 235-248, 2015.

[23] J. T. Betts, Practical methods for optimal control and estimation using nonlinear programming. SIAM, 2010.

[24] R. Bhattacharya, "Optragen: A matlab toolbox for optimal trajectory generation," in Decision and Control, 2006 45th IEEE Conference on. IEEE, 2006, pp. 6832-6836.

[25] P. E. Gill, W. Murray, and M. A. Saunders, "Snopt: An sqp algorithm for large-scale constrained optimization," SIAM review, vol. 47, no. 1, pp. 99-131, 2005.

[26] A. H. Memar and E. T. Esfahani, "Modeling and Dynamic Parameter Identification of the SCHUNK Powerball Robotic Arm," in Volume 5C: 39th Mechanisms and Robotics Conference. ASME, aug 2015, p. V05CT08A024.

[27] S. S. Jujjavarapu, A. H. Memar, and E. T. Esfahani, "Design of a $2 \mathrm{~d}$ haptic system with passive variable stiffness using permanent magnets for upper-limb rehabilitation," in ASME 2017 International Design Engineering Technical Conferences and Computers and Information in Engineering Conference. American Society of Mechanical Engineers, 2017, pp. V003T13A003-V003T13A003.

[28] S. Parastegari, E. Noohi, B. Abbasi, and M. efran, "Failure recovery in robot-human object handover," IEEE Transactions on Robotics, pp. 1-14, 2018. 